\title{
Click chemistry-assisted bioconjugates for
}

\section{hapten immunodiagnostics}

Daniel López-Puertollano, ${ }^{a}$ Consuelo Agulló, ${ }^{\mathrm{a}}$ Josep V. Mercader, ${ }^{\mathrm{b}}$ Antonio Abad-Somovilla, ${ }^{\mathrm{a}}$ Antonio Abad-Fuentes ${ }^{b, *}$

\footnotetext{
a Department of Organic Chemistry, Universitat de València, Doctor Moliner 50, 46100 Burjassot, València, Spain

${ }^{\mathrm{b}}$ Institute of Agrochemistry and Food Technology (IATA), Spanish National Research Council (CSIC), Agustí Escardino 7, 46980 Paterna, València, Spain

* Corresponding author email: aabad@iata.csic.es; tel. +34-963900022; fax +34-963636301.
} 
ABSTRACT

Bioorthogonal reactions have revolutionized the way low molecular weight compounds are coupled to biomolecules. Organic chemistry, polymer science, and chemical biology are among the disciplines that are benefited the most from this breakthrough. Despite the reliability of the click chemistry concept for the efficient and chemoselective functionalization of biomacromolecules with haptens at preferred positions, the fact that azide-alkyne cycloaddition reactions originate new chemical moieties as part of the linker may have delayed their application in the immunodiagnostic field. Using the mycotoxin ochratoxin A as a model compound, we herein demonstrate for the first time that bioconjugates arising from the ligation between an azido-bearing hapten and an alkyne-modified carrier protein are able to elicit the generation of high-affinity monoclonal antibodies suitable for the development of rapid methods for the immunodetection of small organic molecules. 


\section{INTRODUCTION}

Low molecular weight compounds are unable to elicit a secondary, adaptive immune response by their own. For these so-called haptens to trigger the cascade of events that ultimately lead to the proliferation of plasma and memory B cells and to the production of highaffinity IgG antibodies, the compound has to be coupled to an entity carrying epitopes for helper T-cells. ${ }^{1-8}$ Those carriers are most commonly proteins of different origin, like hemocyanins, albumins, globulins, virus-like particles, or toxoids.

The most extended approach for bioconjugation of small compounds is through the formation of stable amide bonds between carboxylate groups of the hapten and amine groups of the protein. $N$-hydroxysuccinimidyl esters prepared by chemistry-mediated carbodiimides are able to efficiently react, under mild conditions, with solvent-accessible lysine residues, which are abundant in most carrier proteins. ${ }^{9-10}$ Very often, total synthetic approaches are required in order to insert the appropriate functionalized linker at the desired position of the molecular framework. Nevertheless, chemical targets occasionally possess a carboxylate group that can be used for direct bioconjugation, like in the case of the mycotoxin ochratoxin $A$, several fluoroquinolone antibiotics, and some medicinal drugs like statins. The strategy of using already available functional groups for coupling, although simple and straightforward, obviates that those moieties are potent antigenic determinants enabling electrostatic interactions and hydrogen bonds between the analyte and amino acid residues in the antibody binding pocket. Accordingly, it looks wise to attach the chemical to the carrier through a different position, so the native carboxylate group is kept unaltered, thus increasing the chance of obtaining specific and high-affinity antibodies. The challenge underlying this approach is the need for protection/deprotection steps in order to introduce a functional group at the selected site of the analyte skeleton. In this respect, bioorthogonal reactions enable the chemoselective covalent coupling of two moieties in the presence of other functionalities. ${ }^{11}$ The prototypical 
example of bioorthogonal reactions in chemical biology is most likely the copper(I)-catalyzed Huisgen 1,3-dipolar azide-alkyne cycloaddition (CuAAC). ${ }^{12-14}$ First codiscovered in 2002 by the groups of Meldal ${ }^{15}$ and Sharpless, ${ }^{16}$ this click chemistry reaction allows the ligation of an azide group and a terminal alkyne group to form a triazole-bridged product under mild, biologically compatible conditions - aqueous solutions, physiological pH, and ambient temperature. ${ }^{17}$ These features, together with the fact that azide and alkyne tags may be easily introduced into small molecules and proteins via chemical synthesis and common in vitro protein modification procedures, have revolutionized bioconjugate chemistry, particularly when $\mathrm{Cu}(\mathrm{I})$-chelating ligands are employed in the coupling reaction.

The main concern arising from using CUAAC reactions for the preparation of bioconjugates intended for vaccination is the presence of the triazole moiety in the linker region connecting the hapten with the protein, because this rigid and aromatic moiety may elicit undesirable antibodies by driving the immune response away from the target compound. Most examples reporting immunization experiments with bioconjugates from click chemistry-mediated approaches concern carbohydrate-based vaccines, either for infectious diseases or for cancer therapeutics. In those studies with rather structurally complex haptens, authors reported that the triazole group is essentially immunosilent. ${ }^{18-23}$ However, when it comes to immunoanalysis of organic chemicals, antibodies with excellent binding properties are strongly desirable because these sorts of analytes are found at very low levels in complex matrices. Accordingly, even minimum disturbances in the antibody-analyte interaction coming from artificially introduced neoepitopes may result in antibodies with insufficient affinity and/or specificity, and therefore unsuitable for the development of rapid analytical tests. In fact, click chemistry approaches have not found wide application in the immunochemistry field yet, and the few reported attempts gave rise to rather disappointing results. Sanders et al. tried to generate antibodies to the mycotoxin deoxynivalenol by introducing a propargyl moiety at the C-8 position of the molecule. ${ }^{24}$ The derivative was coupled to azido-modified bovine serum albumin (BSA), but the 
resulting conjugate provided low titers. Negative results were also obtained by Vasylieva et al. when addressing the generation of antibodies to tetramethylenedisulfotetramine with an azidobearing derivative of this neurotoxin. ${ }^{25}$ Therefore, the question arising is whether bioconjugates from alkyne-azide cycloaddition reactions may be an actually effective approach for the generation of anti-hapten antibodies with the outstanding binding properties that are required in immunodiagnostics or, otherwise, the presence of a rigid aromatic ring in the linker connecting the chemical with the carrier may severely impair that goal. With the aim to shed some light on this issue, we choose the mycotoxin ochratoxin A (OTA) as a model compound. This potent toxic fungal metabolite may be encountered in crops infected by Penicillium and Aspergillus species. ${ }^{26-27}$ The undesirable presence of OTA in food commodities like cereals, raisins, coffee, beer, and wine, constitutes a worldwide problem of public health, so its levels are strictly regulated and controlled. ${ }^{28-30} \mathrm{~A}$ particular chemical feature of OTA is that it bears a carboxylate group. As mentioned above, although this moiety is certainly suitable for coupling to proteins, its capacity to act as a powerful antigenic determinant makes it advisable to link the hapten to the carrier through a different site, and we thought that CuAAC chemistry can greatly assist in facilitating the synthesis of the appropriate derivatives.

\section{RESULTS AND DISCUSSION}

It is well known that the position of the functionalized spacer arm in the hapten may exert a decisive effect on the antibody binding properties. ${ }^{31-35}$ Accordingly, we decided to introduce the aliphatic linker with a terminal azido group at two alternative sites of the OTA structure, i.e., the C-4 position of the benzene ring or the C-3 position of the dihydroisocoumarin ring. The synthetic routes enabling the preparation of the so-called hapten a (with the linker at the aryl 
moiety) and hapten $\mathbf{c}$ (with the linker at the $\mathrm{CH}_{3}$ group) are concisely depicted in Scheme 1 (detailed synthesis procedures can be found in the Supporting Information file).

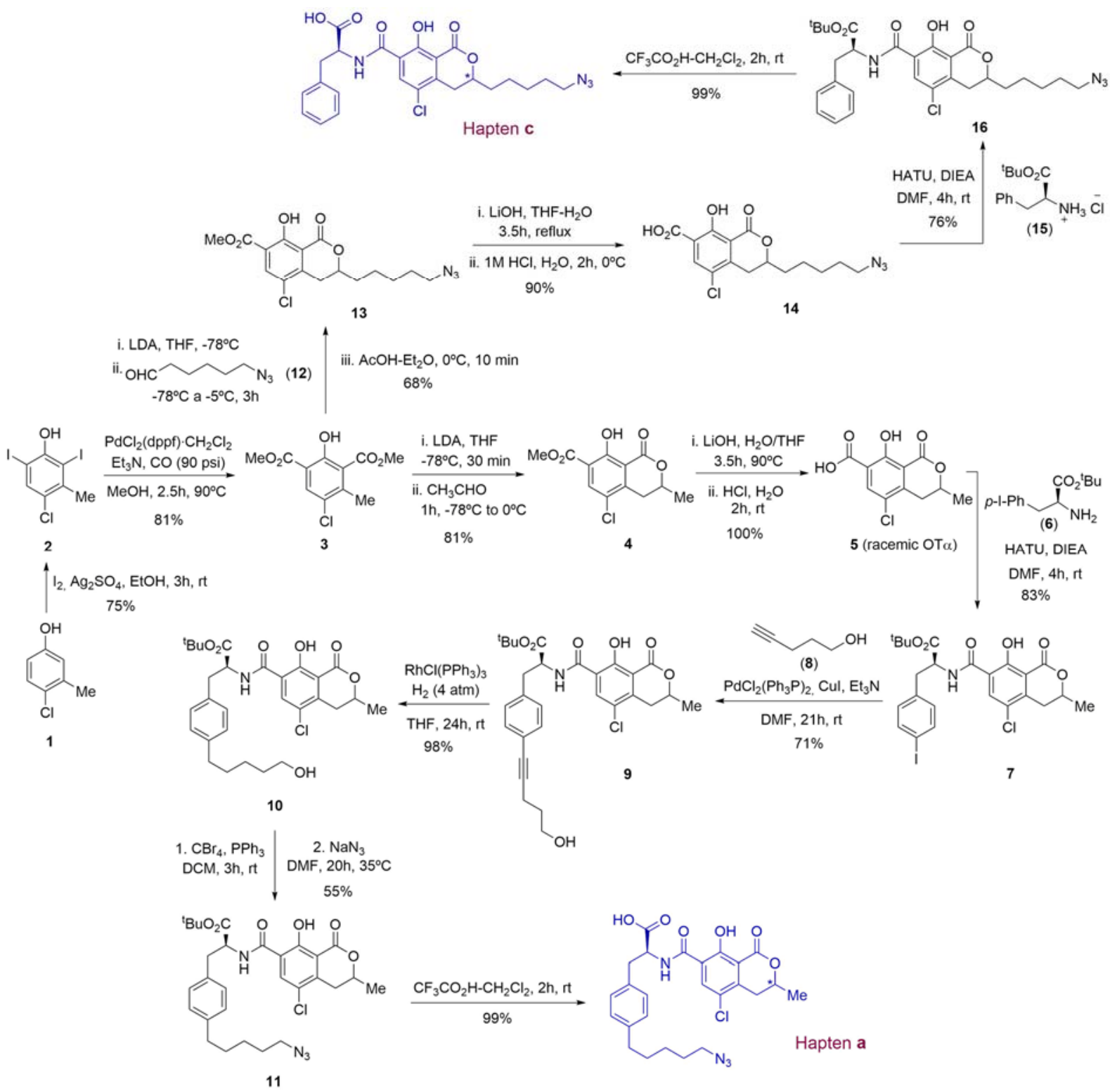

Scheme 1. Synthetic route for the preparation of haptens a and c. Both haptens and all intermediates of their synthesis containing the dihydroisocumarine moiety are 1:1 mixtures of the epimeric diastereomers at the stereogenic center of this moiety (marked with an asterisk in the hapten structures).

Preparation of both haptens followed a similar strategy for the construction of the common OTA core consisting of the preparation of the conveniently functionalized $L$-phenylalanine and dihydroisocoumarin moieties and their coupling through an amide bond. Due to chemical 
compatibility with reactions in the designed synthetic route leading to hapten a, we chose a linker with a terminal azide group for the functionalization of the L-phenylalanine moiety. For synthetic consistency, the same functional group was selected for the preparation of hapten c, so the proteins were accordingly modified with alkyne groups for the subsequent cycloaddition reaction.

Racemic OTa (5) was employed as the starting material for the synthesis of hapten a. Although this chemical is commercially available, we decided to prepare it by an optimized synthetic route previously described by our group. ${ }^{36}$ The rationale behind this decision is that compound $\mathbf{3}$, an intermediate in the pathway leading to 5 , was the key material for the synthesis of hapten $\mathbf{c}$.

The first step in the synthesis of hapten a was the condensation of the dihydroisocoumarin moiety with an iodinated derivative of L-phenylalanine to give $\mathbf{7}$, which was further subjected to a Sonogashira reaction to introduce the linker at the C-4 position of the phenyl ring. After hydrogenation of the acetylenic triple bond of 9 under homogeneous conditions using Wilkinson's catalyst, the hydroxyl group was replaced by the azide group, thus completing the required functionalization of the linker chain in two steps: first, transformation of alcohol $\mathbf{1 0}$ into alkyl halide 11, under Appel's reaction conditions, ${ }^{37}$ followed by substitution of the bromine atom by the highly nucleophilic azide anion. Finally, the desired compound was obtained following deprotection of the native carboxylic group of OTA with trifluoroacetic acid. With this strategy, hapten a was obtained in 9 synthetic steps from 1 with a $15 \%$ overall yield, or in 5 steps with $31 \%$ yield starting from commercially available racemic OT $\alpha$.

The synthesis of hapten c started with the construction of the functionalized dihydroisocoumarin moiety. Metalation of the methyl group of compound $\mathbf{3}$ with lithium diisopropylamide (LDA), followed by reaction of the generated benzylic anion with aldehyde $\mathbf{1 2}$ and acidic work-up, afforded the dihydroisocoumarin derivative $\mathbf{1 3}$, which already incorporated 
the azide-terminal linker for further coupling by click chemistry. Basic hydrolysis of the methyl ester group and condensation reaction between the resulting carboxylic acid $\mathbf{1 4}$ and ( $t$-Boc)protected L-phenylalanine $\mathbf{1 5}$ completed the construction of the hapten skeleton. Finally, as with hapten a, the acidic deprotection of the native carboxylic group of OTA afforded the desired functionalized derivative. This synthetic pathway provided hapten c in 6 steps from 1 with a $28 \%$ overall yield.

With the two haptens at hand, we undertook the modification of the proteins with propargyl groups (Scheme 2). BSA was chosen for the preparation of immunizing bioconjugates, whereas ovalbumin (OVA) and horseradish peroxidase (HRP) were selected for the preparation of the bioconjugates employed in competitive assays - OVA for the antigen-coated ELISA format and HRP for the antibody-coated ELISA format.

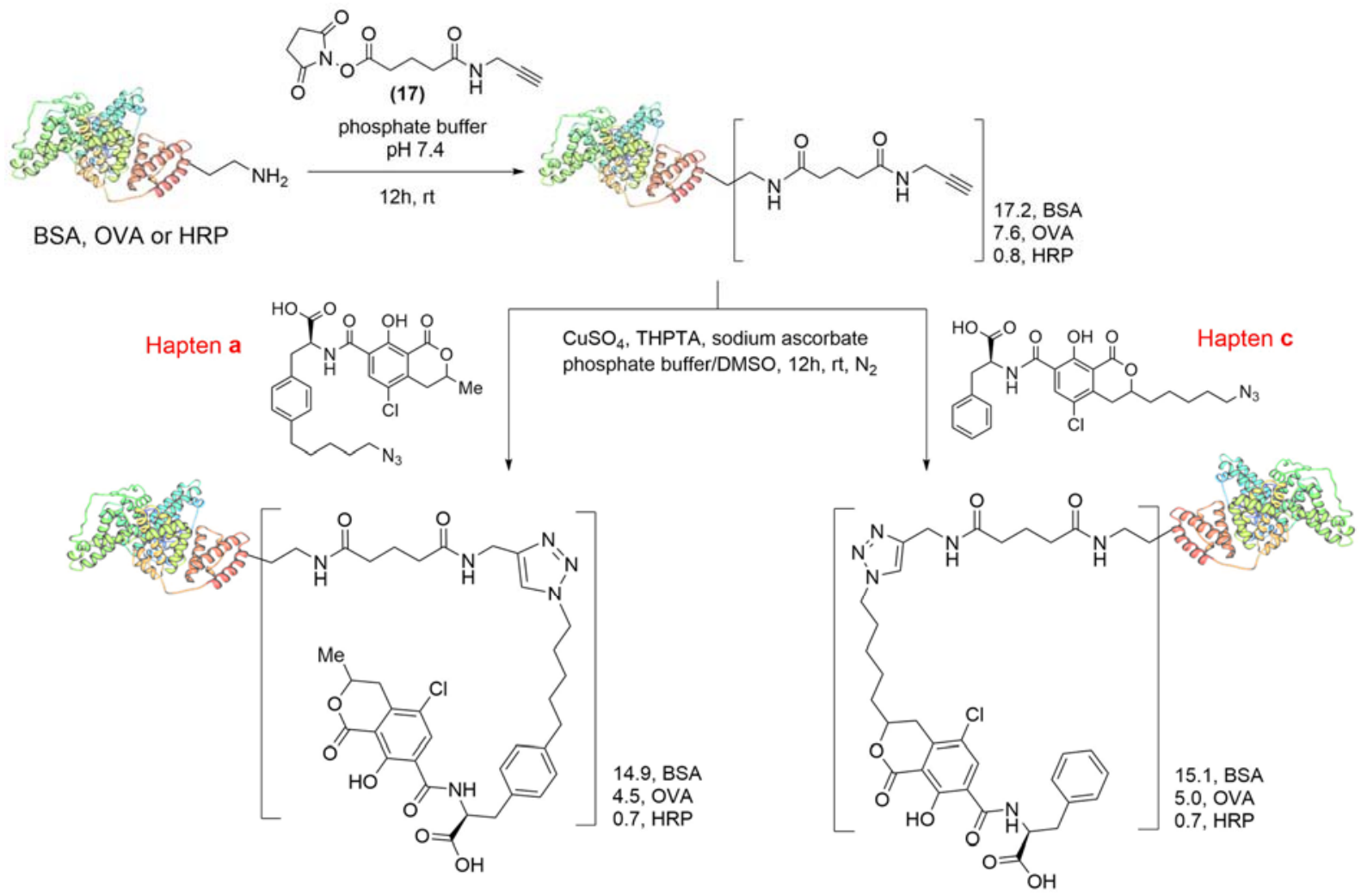

Scheme 2. Preparation of bioconjugates. Proteins were first modified with propargyl groups by reaction with compound 17, and then coupled to haptens by CuAAC reaction. 
Compound $\mathbf{1 7}$ was chosen for the alkynylation of the proteins because it was successfully used by other research groups for the modification of cowpea mosaic virus, poly(lysine) chains, hemocyanin, and albumins. ${ }^{38-42}$ The molar ratio of 17 to BSA in the initial reaction mixture was established at 25 in order to modify a sufficient number of lysine residues as to later achieve a click chemistry-assisted bioconjugate with a rather high hapten loading for immunization. The alkyne-modified BSA was purified by size-exclusion chromatography, and the number of introduced linkers was determined by MALDI-TOF-MS. As shown in Figure 1, the increase in molecular weight with respect to the intact protein (2935 Da) corresponds to an average incorporation of 17.2 alkyne chains into each molecule of BSA.

The CuAAC conditions for the ligation of haptens $\mathbf{a}$ and $\mathbf{c}$ to BSA-alkyne where essentially based on procedures developed by Finn's group. ${ }^{43-44}$ The coupling reaction was carried out with 6 equivalents of hapten per alkyne residue in $100 \mathrm{mM}$ sodium phosphate, $\mathrm{pH} 7.4$, under nitrogen. After $20 \mathrm{~h}$ at room temperature, the bioconjugates were concentrated and purified by size-exclusion chromatography. Hapten-to-protein loadings of 14.9 and 15.1 were found for bioconjugates BSA-a and BSA-c, respectively (Scheme 2), as determined by MALDI-TOF-MS (Figure 1). Concerning OVA and HRP, wherein lower cargos are preferred, initial alkyne-toprotein molar ratios of 12 and 10, respectively, were employed. Under these conditions, 7.6 alkyne residues were introduced into OVA and 0.8 into HRP (Figure S1). After CuAAC reaction under similar conditions to those used with BSA, OVA was modified with an average number of 4.5 and 5.0 residues of hapten a and hapten c, respectively, whereas 0.7 residues of both haptens were introduced into HRP (Scheme 2). 


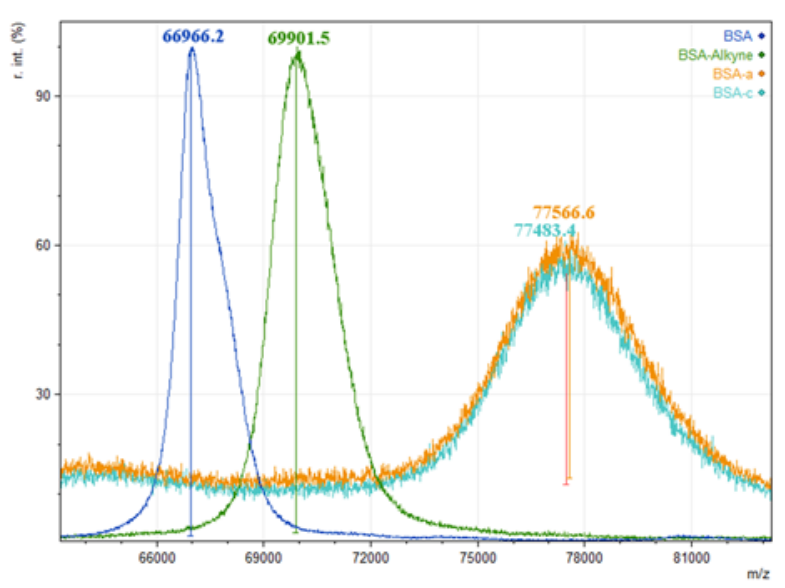

Figure 1. MALDI-TOF mass spectra of BSA (singly charged ions) in their unmodified form (blue), modified with propargyl groups (green), and coupled to haptens a (orange) and c (light blue).

Evaluation of the immune response was first assessed by immunizing two rabbits with each bioconjugate, BSA-a and BSA-c. As judged by antibody-coated ELISA using HRP-a and HRP-c enzyme tracers, excellent immune responses, with titres higher than $3 \times 10^{4}$, were observed. Concerning antiserum capability to recognize OTA in solution, high affinity was observed by competitive ELISA. IC $C_{50}$ values of $9.4 \mathrm{nM}$ and $12.9 \mathrm{nM}$ were found with antisera a\#I and a\#II, respectively, and of $2.1 \mathrm{nM}$ and $0.5 \mathrm{nM}$ with antisera $\mathrm{c \# I}$ and $\mathrm{c \# II,} \mathrm{respectively} \mathrm{(Figure} \mathrm{2).}$

These results clearly prove the suitability of click chemistry-assisted bioconjugates with triazole-bearing linkers for the generation of polyclonal antibodies with excellent binding properties to a small organic compound like OTA. It is also worthy to note that slightly higher affinity to OTA was found with antisera from hapten $\mathbf{c}$, that is, the derivative holding the spacer arm at a distal position from the carboxylic acid moiety of OTA. Regarding specificity, no noticeable recognition of ochratoxin B or ochratoxin C, the two main OTA metabolites, was found - the highest cross-reactivity value was $3.3 \%$ for ochratoxin B with antisera c\#II. This result points out the influence of the chlorine atom and mainly the carboxylic group of OTA in the antibody-antigen interaction. 
Figure 2. Recognition of OTA by polyclonal antibodies from rabbits immunized with bioconjugate BSA-a (blue lines) and BSA-c (black lines). Each line represents the immune response of a different animal. Experimental points by competitive ELISA are the average of three replicates on different days.

At this point of our study, we decided to gain further insights into the participation of the triazole group in the binding event. To this purpose, two haptens previously synthesized by our group bearing carboxylated aliphatic linkers - without the triazole group - at the same positions of the OTA framework than haptens a and $\mathbf{c}$ (Scheme S1) were coupled to HRP. ${ }^{36}$ When these two bioconjugates, so-called HRP-b and HRP-d, were tested by immunoassay, they were perfectly recognized by the antisera from hapten a and antisera from hapten c, respectively, although concentrations slightly higher than those required with the homologous bioconjugates HRP-a and HRP-c were needed in order to attain the same signal. Concerning recognition of OTA, the use of bioconjugates HRP-b and HRP- $\mathbf{d}$ in competitive assays caused a 2-4 times decrease of the IC $5_{50}$ values, thus resulting in better OTA detectability, probably because of the hapten heterology effect. Altogether, these data evidence that, although there is certainly an influence of the triazole moiety in antibody binding, its contribution is of small relevance and it does not handicap the generation of antibodies suitable for immunodiagnostics.

This conclusion was further confirmed by immunizing mice with bioconjugate BSA-c in order to produce monoclonal antibodies (mAbs) by hybridoma technology. From two independent cell 
fusion experiments, 12 hybridoma cell lines producing antibodies with $\mathrm{IC}_{50}$ values to OTA lower than $10 \mathrm{nM}$ were identified. A subset of 5 hybridomas were cloned and stabilized, and the antibodies they produce were comprehensively characterized by both the antigen-coated and the antibody-coated competitive ELISA. Remarkably, mAbs displaying subnanomolar affinity to OTA in both ELISA formats were identified (Table 1). It is also worth mentioning that the affinity to OTA of this group of mAbs was similar to that of the antibodies derived from hapten $\mathbf{d}$ which, as already mentioned, was coupled to BSA through the same position that hapten $\mathbf{c}$ but through NHS ester chemistry instead of click chemistry. In addition, whereas the preparation of hapten d required 10 steps with an overall yield of $18.8 \%$, the synthesis of hapten $\mathbf{c}$ herein reported took 6 steps with an overall yield of $28 \%$. Therefore, bioconjugation through CuAAC chemistry arises as an advantageous approach, even though further studies with other analytes are required to ascertain the scope of this finding.

\begin{tabular}{|c|c|c|c|c|c|c|}
\hline \multirow[b]{2}{*}{$\mathrm{mAb}$} & \multicolumn{3}{|c|}{ Antigen-coated ELISA } & \multicolumn{3}{|c|}{ Antibody-coated ELISA } \\
\hline & {$[\mathrm{mAb}]$} & [OVA-c] & $\mathrm{IC}_{50}$ & {$[\mathrm{mAb}]$} & [HRP-c] & $\mathrm{IC}_{50}$ \\
\hline c\#12 & 30 & 10 & 0.39 & 30 & 10 & 0.31 \\
\hline c\#29 & 30 & 10 & 0.69 & 30 & 10 & 0.57 \\
\hline c\#116 & 30 & 10 & 0.58 & 30 & 10 & 0.85 \\
\hline c\#117 & 100 & 10 & 0.48 & 100 & 10 & 0.29 \\
\hline c\#134 & 30 & 100 & 0.93 & 300 & 10 & 1.13 \\
\hline
\end{tabular}

The $\mathrm{IC}_{50}$ values exhibited by the group of antibodies generated from the bioconjugate BSA-c,

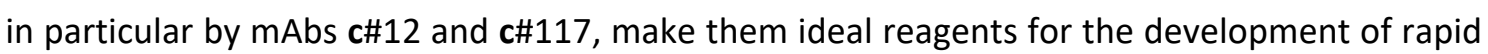
immunoanalytical methods for on-site OTA detection. As a proof-of-concept of this assessment, two immunoassays, an antibody-coated competitive ELISA and a chromatographic immunostrip, were tentatively developed (Table 2). With analytical standards in buffer, the ELISA has a LOQ, defined as the analyte concentration causing a $20 \%$ signal inhibition, of $0.04 \mathrm{ng} / \mathrm{mL}$, a value well below common regulated levels of OTA in foodstuff. As in regular commercial kits, one microtiter 
plate would allow the simultaneous determination of 42 samples and a 6-point standard curve, both in duplicated wells, in 70 min. Regarding immunostrips, signal intensities of the control line (upper stripe) and of the test line (lower stripe) in the membrane visually equals at an OTA concentration of $1-3 \mathrm{ng} / \mathrm{mL}$, while a common desktop scanner for reading signal intensities enable to quantify OTA at levels higher than $0.04 \mathrm{ng} / \mathrm{mL}$ by using the ratio between the signal of both lines.

Table 2. Analytical parameters of the developed immunoanalytical methods for OTA ${ }^{\mathrm{a}}$.

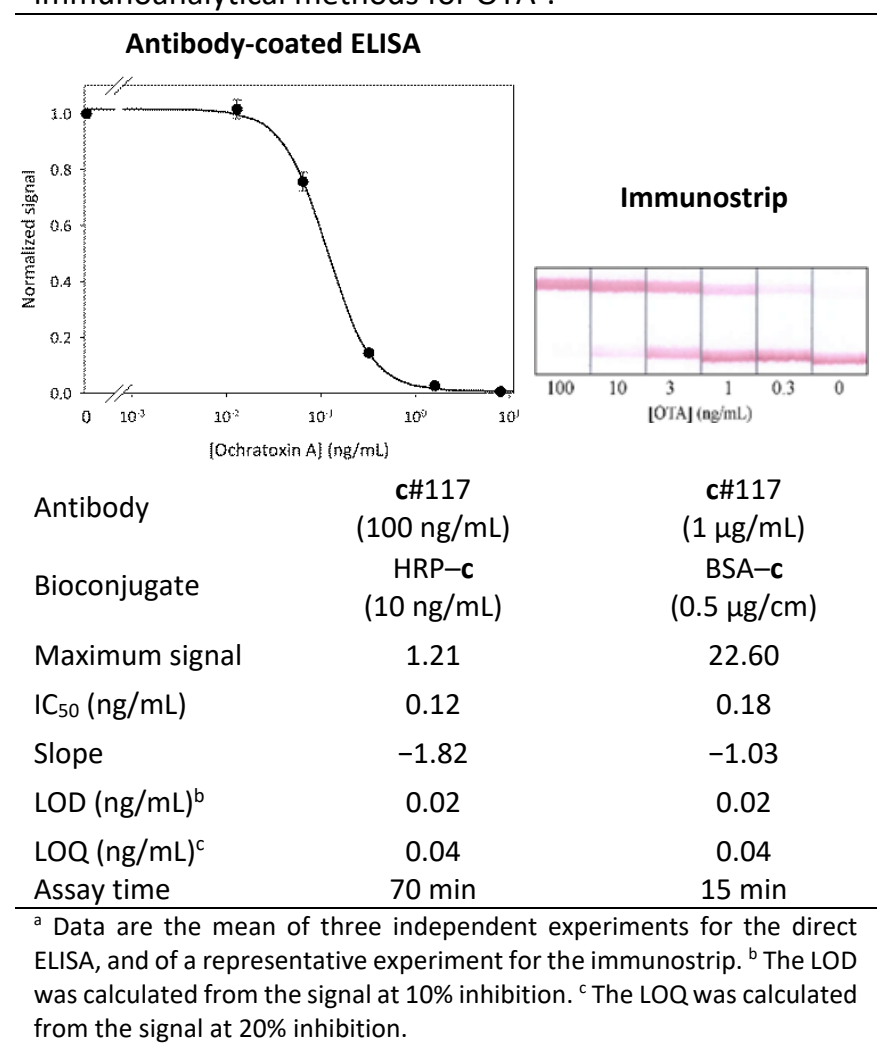

CONCLUSIONS

This study demonstrates for the first time, using OTA as a model compound, that CUAAC chemistry is a convenient approach for the preparation of protein-hapten bioconjugates intended for the generation of suitable antibodies for immunodiagnostics of small organic 
chemicals. Our results point out that the participation of the triazole moiety in antibody binding is not as critical as it could be thought, so its presence in the spacer arm does not preclude the production of antibodies with apparent affinity constants for the analyte in the subnanomolar range. In our opinion, this finding opens the door to obtaining highly valuable biotechnological immunoreagents for other relevant compounds, like antibiotics, biotoxins, and drugs, through unexplored chemical approaches involving innovative positions for linker attachment and hapten functionalization. Further studies should certainly contribute to clarify the generalizability of the herein reported strategy.

\section{EXPERIMENTAL PROCEDURES}

\section{Preparation of click chemistry-assisted bioconjugates.}

Modification of proteins with propargyl groups. Compound 17 was dissolved in $N, N^{\prime}-$ dimethylformamide (DMF) and drop wise added to a solution of protein in phosphate buffer (PB, pH 7.4). The conjugation reaction was carried out with a DMF maximum content of $10 \%$ $(v / v)$. After stirring overnight at room temperature, the protein conjugates were purified by sizeexclusion chromatography using PB as eluent at a flow rate of $5 \mathrm{~mL} / \mathrm{min}$.

Coupling of haptens to alkyne-modified proteins by CUAAC reaction. Solutions of haptens a and $\mathbf{c}$ in dimethylsulphoxide were drop wise added to alkyne-modified protein solutions in PB, $\mathrm{pH}$ 7.4. After adding a premixed solution of THPTA/Cu(I) complex $(5: 1)$ in Milli-Q $\mathrm{H}_{2} \mathrm{O}$, the mixture was purged by repeated vacuum-nitrogen cycles. Finally, a solution of sodium ascorbate in Milli-Q $\mathrm{H}_{2} \mathrm{O}$ was added and the final reaction volume was adjusted with PB, $\mathrm{pH}$ 7.4, followed by vacuum-nitrogen cycles. The solutions were incubated $20 \mathrm{~h}$ at room temperature, and the reaction was then stopped using Amicon Ultra $410 \mathrm{~K}$ centrifugal filters. The protein conjugates were purified by size-exclusion chromatography. 
MALDI-TOF mass spectrometry analysis of modified proteins. $100 \mu \mathrm{L}$ of native protein, alkynemodified protein or protein-hapten bioconjugate $(0.5-1 \mathrm{mg} / \mathrm{mL})$ was dialyzed against Milli- $\mathrm{Q}$ water and then lyophilized. The samples were dissolved in Milli-Q $\mathrm{H}_{2} \mathrm{O}$ to a final concentration of $1 \mu \mathrm{g} / \mu \mathrm{L}$. Then, $1 \mu \mathrm{L}$ of every sample solution was spotted onto the MALDI plate. After the droplets were air dried at room temperature, $1 \mu \mathrm{L}$ of matrix $[10 \mathrm{mg} / \mathrm{mL}$ sinapinic acid in $0.1 \%$ trifluoroacetic acid- $\left.\mathrm{CH}_{3} \mathrm{CN} / \mathrm{H}_{2} \mathrm{O}(7: 3 \mathrm{v} / \mathrm{v})\right]$ was added and allowed to air-dry at room temperature. Samples were analysed in a 5800 MALDI TOF/TOF (ABSciex) in positive linear mode (1500 shots every position) in a mass range of $15000-100000 \mathrm{~m} / \mathrm{z}$. Previously, the plate was calibrated with $1 \mu \mathrm{L}$ of the TOF/TOF calibration mixture (ABSciex), in 13 positions. Every sample was calibrated by 'close external calibration' method with a BSA, OVA, or HRP spectrum acquired in a close position.

\section{Antibody generation}

Female New Zealand white rabbits were immunized at 21-day intervals by subcutaneous injection with $0.3 \mathrm{mg}$ of BSA-hapten conjugate in $1 \mathrm{~mL}$ of a 1:1 emulsion between sterile 100 $\mathrm{mM}$ phosphate buffer, $\mathrm{pH} 7.4$, and Freund's adjuvant (complete for the first dose and incomplete for subsequent boosts). Ten days after the fourth injection, rabbits were exsanguinated by intracardiac puncture. Blood samples were allowed to coagulate overnight at $4{ }^{\circ} \mathrm{C}$, and sera were separated by centrifugation (3000×g, $\left.20 \mathrm{~min}\right)$. Finally, antibodies were precipitated with one volume of saturated ammonium sulphate solution. Salting out was performed twice, and precipitates were stored at $4{ }^{\circ} \mathrm{C}$.

Two-month old female Balb/c mice were immunized by intraperitoneal injection of $0.1 \mathrm{mg}$ of BSA-hapten conjugate in $200 \mu \mathrm{L}$ of a water-in-oil emulsion that was prepared using Freund's adjuvants as described above. Three doses were applied at 21-day intervals, and a fourth injection in sterile PBS with the same amount of bioconjugate was given at least 21 days after the third injection and 4 days before the animal was sacrificed. Hybridomas were prepared by 
fusion of mouse myeloma cells with splenocytes from two equally-immunized mice following standard procedures. ${ }^{45}$ Antibody-producing cells were identified by a double screening procedure. Twelve days after cell fusion, hybridoma culture supernatants were first screened by differential antigen-coated competitive ELISA on microtiter plates coated with $0.1 \mu \mathrm{g} / \mathrm{mL}(100$ $\mu \mathrm{L}$ per well) of the homologous OVA-hapten bioconjugate. Fifty microliter of each supernatant was added to two adjacent wells of an ELISA plate, one containing $50 \mu \mathrm{L}$ of PBS (blank) and the other one containing $50 \mu \mathrm{L}$ of $200 \mathrm{nM}$ OTA in PBS. The ratio between the signals of both wells was used as the criterion for selecting the antibodies with the highest affinity. Fresh culture medium was added to those culture plate wells performing best, and next day they were reevaluated by checkerboard competitive ELISA. This second screening assay was carried out with two coating concentrations of the homologous OVA-hapten conjugate $(0.01$ and $0.1 \mu \mathrm{g} / \mathrm{mL})$, four supernatant dilutions $(1 / 10,1 / 50,1 / 250$, and $1 / 1250)$, and three OTA levels $(0,10$, and 100 $n M)$.

\section{Competitive ELISA}

Antibody-coated direct format. 96-well plates were coated with $100 \mu \mathrm{L}$ per well of capture antibody [goat anti-mouse for monoclonal antibodies (GAM) and goat anti-rabbit for polyclonal antibodies (GAR)] diluted in $50 \mathrm{mM}$ carbonate-bicarbonate buffer, $\mathrm{pH}$ 9.6, by overnight incubation at $4{ }^{\circ} \mathrm{C}$. Next day, plates were washed four times with washing solution (150 mM $\mathrm{NaCl}$ containing $0.05 \%(\mathrm{v} / \mathrm{v})$ Tween 20), anti-OTA antibodies in PBST were added (100 $\mu \mathrm{L}$ per well), and plates were incubated for $1 \mathrm{~h}$ at room temperature. After washing as described before, the competitive step was carried out by mixing $50 \mu \mathrm{L}$ per well of OTA solution in PBS and $50 \mu \mathrm{L}$ per well of HRP-hapten tracer in PBST. After $1 \mathrm{~h}$ incubation at room temperature and washing, signal was generated by adding $100 \mu \mathrm{L}$ per well of enzyme substrate solution $[2 \mathrm{mg} / \mathrm{mL}$ o-phenylendiamine in $25 \mathrm{mM}$ citrate and $62 \mathrm{mM}$ phosphate buffer, $\mathrm{pH} 5.4$, containing $0.012 \%$ (v/v) H2O2] and incubation during 10 min at room temperature. Finally, the enzymatic reaction 
was stopped with $100 \mu \mathrm{L}$ per well of $1 \mathrm{M} \mathrm{H}_{2} \mathrm{SO}_{4}$, and the absorbance was immediately read at $492 \mathrm{~nm}$ using the absorbance at $650 \mathrm{~nm}$ as a reference value.

Antigen-coated indirect format. Microplates were coated with $100 \mu \mathrm{L}$ per well of OVAhapten bioconjugate solution in $50 \mathrm{mM}$ carbonate-bicarbonate buffer, $\mathrm{pH}$ 9.6. After overnight incubation at room temperature, plates were washed four times with washing solution. The competitive step was carried out during $1 \mathrm{~h}$ at room temperature with $50 \mu \mathrm{L}$ per well of OTA solution in PBS plus $50 \mu \mathrm{L}$ per well of antibody dilution in PBST. After washing, $100 \mu \mathrm{L}$ per well of HRP-labelled secondary antibody in PBST was added, and the plates were incubated at room temperature for $1 \mathrm{~h}$. Finally, microwells were washed as before and the signal was obtained as described for direct assays.

\section{Competitive LFIA protocol}

Preparation of AuNPGAM@mAb. Commercial 40-nm gold nanoparticles coated with GAM (OD $=10)$ were diluted ten times in $10 \mathrm{mM}$ HEPES buffer ( $\mathrm{pH} 7.4)$. Monoclonal antibody was added to the desired concentration from a stock solution in 10 mM HEPES buffer $(\mathrm{pH} 8.0)$ and the mixture was incubated for $1 \mathrm{~h}$ at room temperature. After addition of Tween20 to obtain a final concentration of $0.05 \%(\mathrm{v} / \mathrm{v})$, nanoparticles were stored at $4{ }^{\circ} \mathrm{C}$.

Preparation of immunostrips. Solutions of GAM (control line) and BSA-hapten bioconjugate (test line) in $100 \mathrm{mM}$ phosphate buffer containing $150 \mathrm{mM} \mathrm{NaCl}, \mathrm{pH} 7.4$, were dispensed on a nitrocellulose membrane $(300 \times 25 \mathrm{~mm})$ at a flow rate of $0.5 \mu \mathrm{L} / \mathrm{cm}$ using a BioDot ZX1010 machine. BSA-hapten bioconjugate and GAM solution were dispensed at $10 \mathrm{~mm}$ and $15 \mathrm{~mm}$ from the base of the membrane, respectively. Membranes were dried at room temperature for $1 \mathrm{~h}$ and were placed $15 \mathrm{~mm}$ from the base of the backing card $(300 \times 78 \mathrm{~mm})$. Nitrocellulose sample pad $(300 \times 17 \mathrm{~mm})$ and adsorbent pad $(300 \times 43 \mathrm{~mm})$ were assembled overlapping $2 \mathrm{~mm}$ and $3 \mathrm{~mm}$, respectively, with the membrane. 
Assay protocol. Dipstick lateral flow immunoassays were carried out in $300-\mu \mathrm{L}$ wells by mixing $12 \mu \mathrm{L}$ of the suspension of antibody-coated gold nanoparticles and $88 \mu \mathrm{L}$ of OTA solution in 100 $\mathrm{mM}$ TRIS buffer containing $30 \mathrm{mM} \mathrm{NaCl}, \mathrm{pH}$ 8.0. The mixtures were incubated $5 \mathrm{~min}$ at room temperature and then the immunostrips were plunged into the well. After incubating the immunostrips in vertical position for $10 \mathrm{~min}$, sample pads were removed, the membranes were dried for 3 min under a cold airflow, and signals were registered with a desktop scanner at a resolution of $1200 \mathrm{dpi}$.

\section{ACKNOWLEDGMENTS}

This work was supported by the Spanish Ministerio de Economía y Competitividad (AGL201564488 and RTI2018-096121), Conselleria d’Educació, Investigació, Cultura i Esport de la Generalitat Valenciana (AICO/2018/111), and cofinanced by European Regional Development Funds. D.L.-P. was supported by a FPU predoctoral grant (FPU14/05266) from the Spanish Ministerio de Educación, Cultura y Deporte. The proteomic analysis was performed at the Proteomics Section of SCSIE of the University of Valencia which belongs to ProteoRed, PRB2-3, and was supported by grant PT17/0019, of the PE I+D+i 2013-2016, and funded by ISCIII and ERDF. The technical assistance by Paula Peña Murgui is greatly appreciated. Animal manipulation was carried out at the Animal Production Section, also belonging to the SCSIE of the University of Valencia.

Samples of the immunoreagents herein reported are available upon request from the corresponding authors.

\section{ASSOCIATED CONTENT}

\section{Supporting information}


General reagents and equipment, detailed synthesis procedures, NMR spectra, and mass spectrometry data. This material is available free of charge via the Internet at http://pubs.acs.org.

\section{REFERENCES}

(1) Ramachandra, L., Simmons, D., Harding, C. V. (2009) MHC molecules and microbial antigen processing in phagosomes. Curr. Opin. Immunol. 21, 98-104.

(2) Burgdorf, S., Kurts, C. (2008) Endocytosis mechanisms and the cell biology of antigen presentation. Curr. Opin. Immunol. 20, 89-95.

(3) Yoshida, T., Mei, H., Doerner, T., Hiepe, F., Radbruch, A., Fillatreau, S., Hoyer, B. F. (2010) Memory B and memory plasma cells. Immunol. Rev. 237, 117-139.

(4) Bachmann, M. F., Jennings, G. T. (2010) Vaccine delivery: a matter of size, geometry, kinetics and molecular patterns. Nat. Rev. Immunol. 10, 787-96.

(5) Costantino, P., Rappuoli, R., Berti, F. (2011) The design of semi-synthetic and synthetic glycoconjugate vaccines. Expert Opin. Drug Dis. 6, 1045-1066.

(6) Yin, Z., Comellas-Aragonés, M., Chowdhury, S., Bentley, P., Kaczanowska, K., Benmohamed, L., Gildersleeve, J. C., Finn, M. G., Huang, X. (2013) Boosting immunity to small tumor-associated carbohydrates with bacteriophage qbeta capsids. ACS Chem. Biol. 8, 12531262.

(7) Ceballos-Alcantarilla, E., Abad-Somovilla, A., Agulló, C., Abad-Fuentes, A., Mercader, J. V. (2017) Protein-free hapten-carbon nanotube constructs induce the secondary immune response. Bioconjug. Chem. 28, 1630-1638.

(8) Parra, J., Abad-Somovilla, A., Mercader, J. V., Taton, T. A., Abad-Fuentes, A. (2013) Carbon nanotube-protein carriers enhance size-dependent self-adjuvant antibody response to haptens. J. Control Release 170, 242-251.

(9) Pickens, C. J., Johnson, S. N., Pressnall, M. M., Leon, M. A., Berkland, C. J. (2018)

Practical considerations, challenges, and limitations of bioconjugation via azide-alkyne cycloaddition. Bioconjug. Chem. 29, 686-701.

(10) Elahipanah, S., O'Brien, P. J., Rogozhnikov, D., Yousaf, M. N. (2017) General dialdehyde click chemistry for amine bioconjugation. Bioconjug. Chem. 28, 1422-1433.

(11) Thirumurugan, P., Matosiuk, D., Jozwiak, K. (2013) Click chemistry for drug development and diverse chemical-biology applications. Chem. Rev. 113, 4905-4979. 
(12) Dirks, A. J., Cornelissen, J. J. L. M., Nolte, R. J. M. (2009) Monitoring protein-polymer conjugation by a fluorogenic $\mathrm{Cu}(\mathrm{I})$-catalyzed azide-alkyne 1,3-dipolar cycloaddition. Bioconjug. Chem. 20, 1129-1138.

(13) Best, M. D. (2009) Click chemistry and bioorthogonal reactions: unprecedented selectivity in the labeling of biological molecules. Biochemistry 48, 6571-6584.

(14) Lallana, E., Riguera, R., Fernández-Megía, E. (2011) Reliable and efficient procedures for the conjugation of biomolecules through Huisgen azide-alkyne cycloadditions. Angew. Chem. Int. Ed. Engl. 50, 8794-8804.

(15) Tornoe, C. W., Christensen, C., Meldal, M. (2002) Peptidotriazoles on solid phase: $[1,2,3]$-triazoles by regiospecific copper(i)-catalyzed 1,3-dipolar cycloadditions of terminal alkynes to azides. J. Org. Chem. 67, 3057-3064.

(16) Rostovtsev, V. V., Green, L. G., Fokin, V. V., Sharpless, K. B. (2002) A stepwise Huisgen cycloaddition process: copper(I)-catalyzed regioselective "ligation" of azides and terminal alkynes. Angew. Chem. Int. Ed. Engl. 41, 2596-2599.

(17) Palomo, J. M. (2012) Click reactions in protein chemistry: from the preparation of semisynthetic enzymes to new click enzymes. Org. Biomol. Chem. 10, 9309-9318.

(18) Adamo, R., Hu, Q.-Y., Torosantucci, A., Crotti, S., Brogioni, G., Allan, M., Chiani, P., Bromuro, C., Quinn, D., Tontini, M., et. al. (2014) Deciphering the structure-immunogenicity relationship of anti-Candida glycoconjugate vaccines. Chem. Sci. 5, 4302-4311.

(19) Lipinski, T., Luu, T., Kitov, P. I., Szpacenko, A., Bundle, D. R. (2011) A structurally diversified linker enhances the immune response to a small carbohydrate hapten. Glycoconj. J. $28,149-164$.

(20) Yin, Z., Nguyen, H. G., Chowdhury, S., Bentley, P., Bruckman, M. A., Miermont, A., Gildersleeve, J. C., Wang, Q., Huang, X. (2012) Tobacco mosaic virus as a new carrier for tumor associated carbohydrate antigens. Bioconjug. Chem. 23, 1694-1703.

(21) Hu, Q.-Y., Allan, M., Adamo, R., Quinn, D., Zhai, H., Wu, G., Clark, K., Zhou, J., Ortiz, S., Wang, B., et. al. (2013) Synthesis of a well-defined glycoconjugate vaccine by a tyrosineselective conjugation strategy. Chem. Sci. 4, 3827-3832.

(22) Cai, H., Shukla, S., Wang, C., Masarapu, H., Steinmetz, N. F. (2019) Heterologous primeboost enhances the antitumor immune response elicited by plant-virus-based cancer vaccine. J. Am. Chem. So.c 141, 6509-6518.

(23) Nilo, A., Morelli, L., Passalacqua, I., Brogioni, B., Allan, M., Carboni, F., Pezzicoli, A., Zerbini, F., Maione, D., Fabbrini, M., et al. (2015) Anti-group B Streptococcus glycan-conjugate vaccines using pilus protein GBS80 as carrier and antigen: comparing lysine and yyrosinedirected conjugation. ACS Chem. Biol. 10, 1737-1746. 
(24) Sanders, M., Guo, Y., Lyer, A., García, Y. R., Galvita, A., Heyerick, A., Deforce, D., Risseeuw, M. D., Van Calenbergh, S., Bracke, M., et. al. (2014) An immunogen synthesis strategy for the development of specific anti-deoxynivalenol monoclonal antibodies. Food Addit. Contam. Part A Chem. Anal. Control Expo. Risk Assess. 31, 1751-1759.

(25) Vasylieva, N., Barnych, B., Rand, A., Inceoglu, B., Gee, S. J., Hammock, B. D. (2017) sensitive immunoassay for detection and quantification of the neurotoxin, tetramethylenedisulfotetramine. Anal. Chem. 89, 5612-5619.

(26) Heussner, A. H., Bingle, L. E. (2015) Comparative ochratoxin toxicity: a review of the available data. Toxins (Basel) 7, 4253-4282.

(27) Tao, Y., Xie, S., Xu, F., Liu, A., Wang, Y., Chen, D., Pan, Y., Huang, L., Peng, D., Wang, X., et al. (2018) Ochratoxin A: toxicity, oxidative stress and metabolism. Food Chem. Toxicol. 112, $320-331$.

(28) Bellver Soto, J., Fernández-Franzón, M., Ruiz, M. J., Juan-García, A. (2014) Presence of ochratoxin A (OTA) mycotoxin in alcoholic drinks from southern European countries: wine and beer. J. Agric. Food Chem. 62, 7643-7651.

(29) Amézqueta, S., Schorr-Galindo, S., Murillo-Arbizu, M., González-Peñas, E., López de Cerain, A., Guiraud, J. P. (2012) OTA-producing fungi in foodstuffs: a review. Food Control 26, 259-268.

(30) Bertuzzi, T., Rastelli, S., Mulazzi, A., Donadini, G., Pietri, A. (2011) Mycotoxin occurrence in beer produced in several European countries. Food Control 22, 2059-2064.

(31) Quiñones-Reyes, G., Agulló, C., Mercader, J. V., Abad-Somovilla, A., Abad-Fuentes, A. (2019) Synthetic haptens and monoclonal antibodies to the cyanotoxin anatoxin-a. Angew. Chem. Int. Ed. Engl. 58, 9134-9139.

(32) Mercader, J. V., Agulló, C., Abad-Somovilla, A., Abad-Fuentes, A. (2011) Synthesis of site-heterologous haptens for high-affinity anti-pyraclostrobin antibody generation. Org. Biomol. Chem. 9, 1443-1453.

(33) Parra, J., Mercader, J. V., Agulló, C., Abad-Somovilla, A., Abad-Fuentes, A. (2012) Generation of anti-azoxystrobin monoclonal antibodies from regioisomeric haptens functionalized at selected sites and development of indirect competitive immunoassays. Anal. Chim. Acta 715, 105-112.

(34) Esteve-Turrillas, F. A., Agulló, C., Mercader, J. V., Abad-Somovilla, A., Abad-Fuentes, A. (2018) Rationally designed haptens for highly sensitive monoclonal antibody-based immunoanalysis of fenhexamid. Analyst 143, 4057-4066. 
(35) Ceballos-Alcantarilla, E., López-Puertollano, D., Agulló, C., Abad-Fuentes, A., AbadSomovilla, A., Mercader, J. V. (2018) Combined heterologies for monoclonal antibody-based immunoanalysis of fluxapyroxad. Analyst 143, 5718-5727.

(36) López-Puertollano, D., Mercader, J. V., Agulló, C., Abad-Somovilla, A., Abad-Fuentes, A. (2018) Novel haptens and monoclonal antibodies with subnanomolar affinity for a classical analytical target, ochratoxin A. Sci. Rep. 8, 9761.

(37) van Kalkeren, H. A., van Delft, F. L., Rutjes, F. P. J. T. (2012) Catalytic Appel reactions. Pure Appl. Chem. 85, 817-828.

(38) Wang, Q., Chan, T. R., Hilgraf, R., Fokin, V. V., Sharpless, K. B., Finn, M. G. (2003) Bioconjugation by copper(I)-catalyzed azide-alkyne [3+2] cycloaddition. J. Am. Chem. Soc. 125, 3192-3193.

(39) Aime, S., Dastru, W., Gobetto, R., Viale, A. (2005) para-Hydrogenation of unsaturated moieties on poly(lysine) derived substrates for the development of novel hyperpolarized MRI contrast agents. Org. Biomol. Chem. 3, 3948-3954.

(40) Kaltgrad, E., Gupta, S., Punna, S., Huang, C. Y., Chang, A., Wong, C. H., Finn, M. G., Blixt, O. (2007) Anti-carbohydrate antibodies elicited by polyvalent display on a viral scaffold. ChemBioChem 8, 1455-1462.

(41) Shi, W., Dolai, S., Averick, S., Fernando, S. S., Saltos, J. A., L'Amoreaux, W., Banerjee, P., Raja, K. (2009) A general methodology toward drug/dye incorporated living copolymer-protein hybrids: (NIRF dye-glucose) copolymer-avidin/BSA conjugates as prototypes. Bioconj. Chem. $20,1595-1601$.

(42) Lipinski, T., Kitov, P. I., Szpacenko, A., Paszkiewicz, E., Bundle, D. R. (2011) Synthesis and immunogenicity of a glycopolymer conjugate. Bioconjug. Chem. 22, 274-281.

(43) Hong, V., Presolski, S. I., Ma, C., Finn, M. G. (2209) Analysis and optimization of coppercatalyzed azide-alkyne cycloaddition for bioconjugation. Angew. Chem. Int. Ed. Engl. 48, 9879-9883.

(44) Presolski, S. I., Hong, V. P., Finn, M. G. (2011) Copper-catalyzed azide-alkyne click chemistry for bioconjugation. Curr. Proto.c Chem. Biol. 3, 153-162.

(45) Mercader, J. V., Suárez-Pantaleón, C., Agulló, C., Abad-Somovilla, A., Abad-Fuentes, A. (2008) Production and characterization of monoclonal antibodies specific to the strobilurin pesticide pyraclostrobin. J. Agric. Food Chem. 56, 7682-7690. 

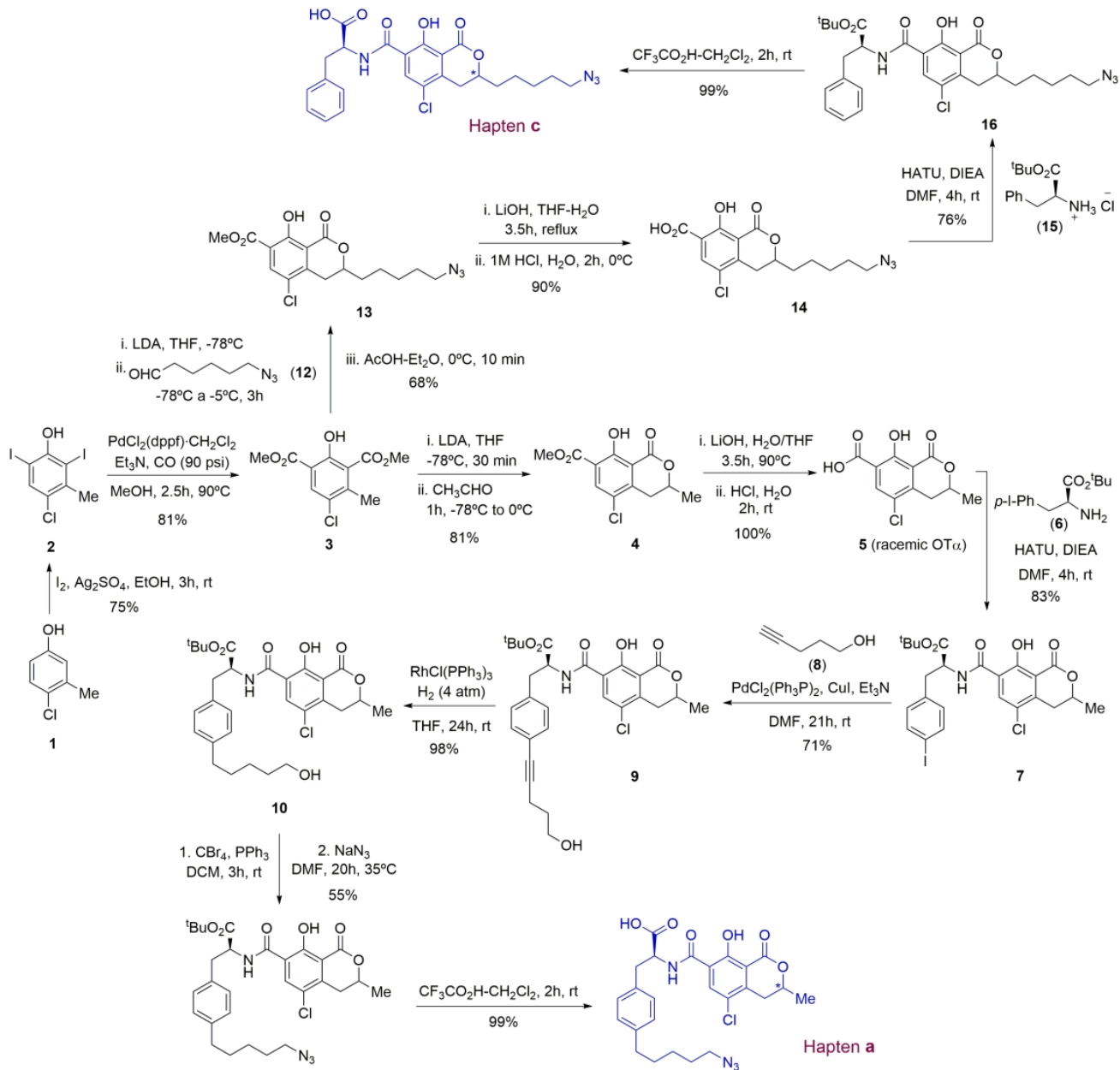

Scheme 1. Synthetic route for the preparation of haptens a and c. Both haptens and all intermediates of their synthesis containing the dihydroisocumarine moiety are 1:1 mixtures of the epimeric diastereomers at the stereogenic center of this moiety (marked with an asterisk in the hapten structures).

$266 \times 261 \mathrm{~mm}(300 \times 300 \mathrm{DPI})$ 

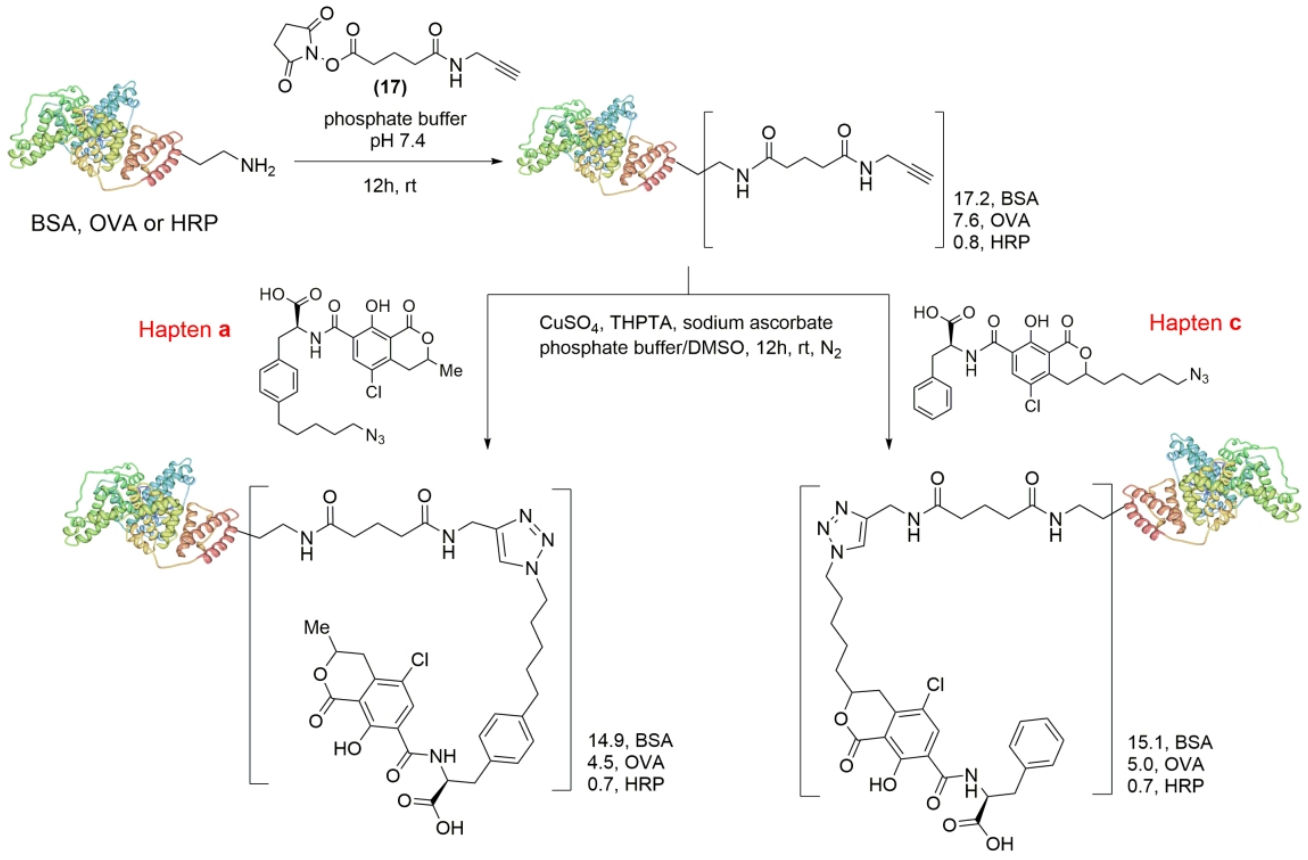

Scheme 2. Preparation of bioconjugates. Proteins were first modified with propargyl groups and then coupled to haptens by CuAAC reaction

$241 \times 158 \mathrm{~mm}(300 \times 300 \mathrm{DPI})$ 
1

2

3

4

5

6

7

8

9

10

11

12

13

14

15

16

17

18

19

20

21

22

23

24

25

26

27

28

29

30

31

32

33

34

35

36

37

38

39

40

41

42

43

44

45

46

47

48

49

50

51

52

53

54

55

56

57

58

59

60

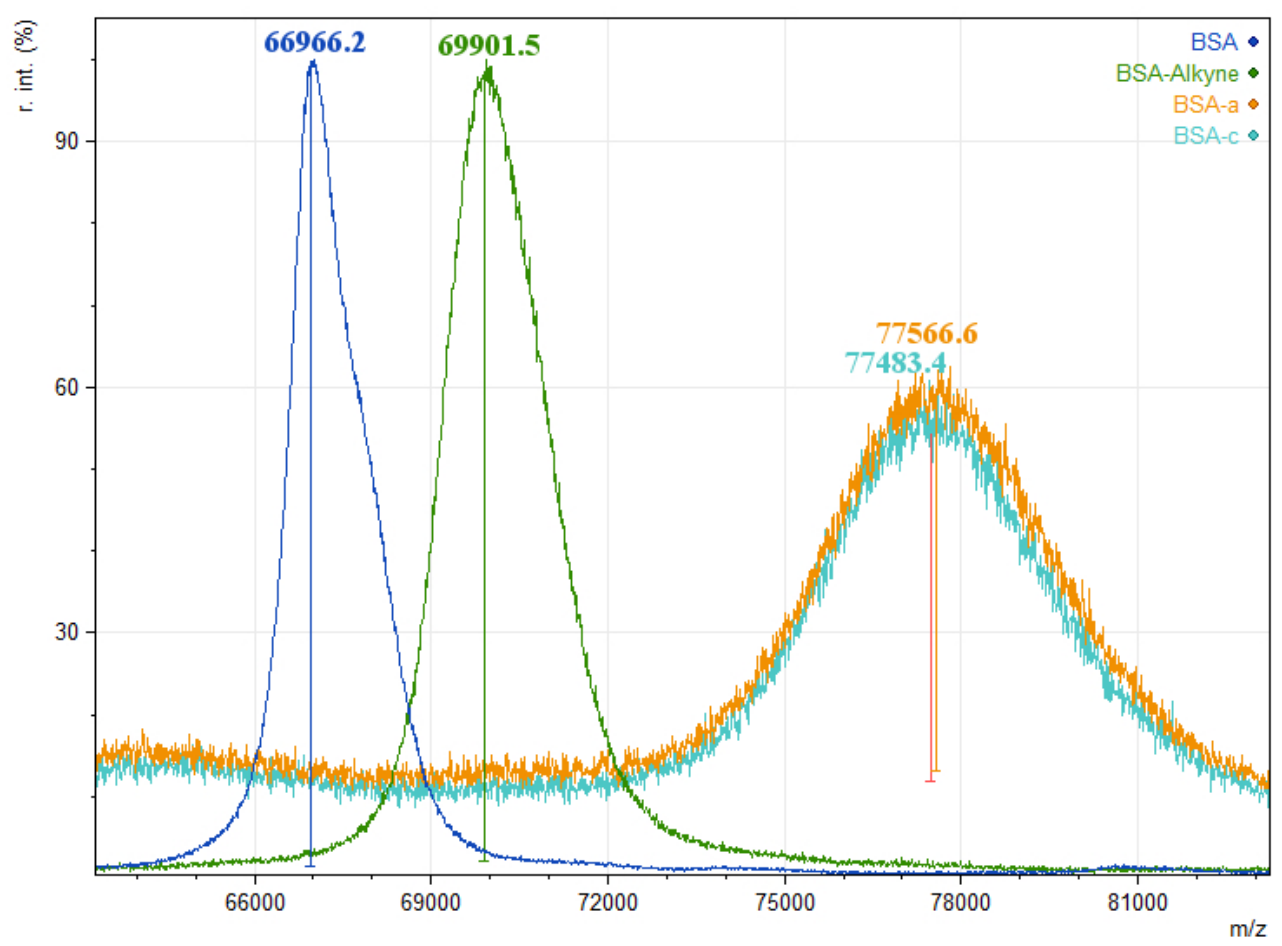

Figure 1. MALDI-TOF mass spectra of BSA (singly charged ions) in their unmodified form (blue), modified with propargyl groups (green), and coupled to haptens a (orange) and c (light blue).

$132 \times 97 \mathrm{~mm}(144 \times 144 \mathrm{DPI})$ 


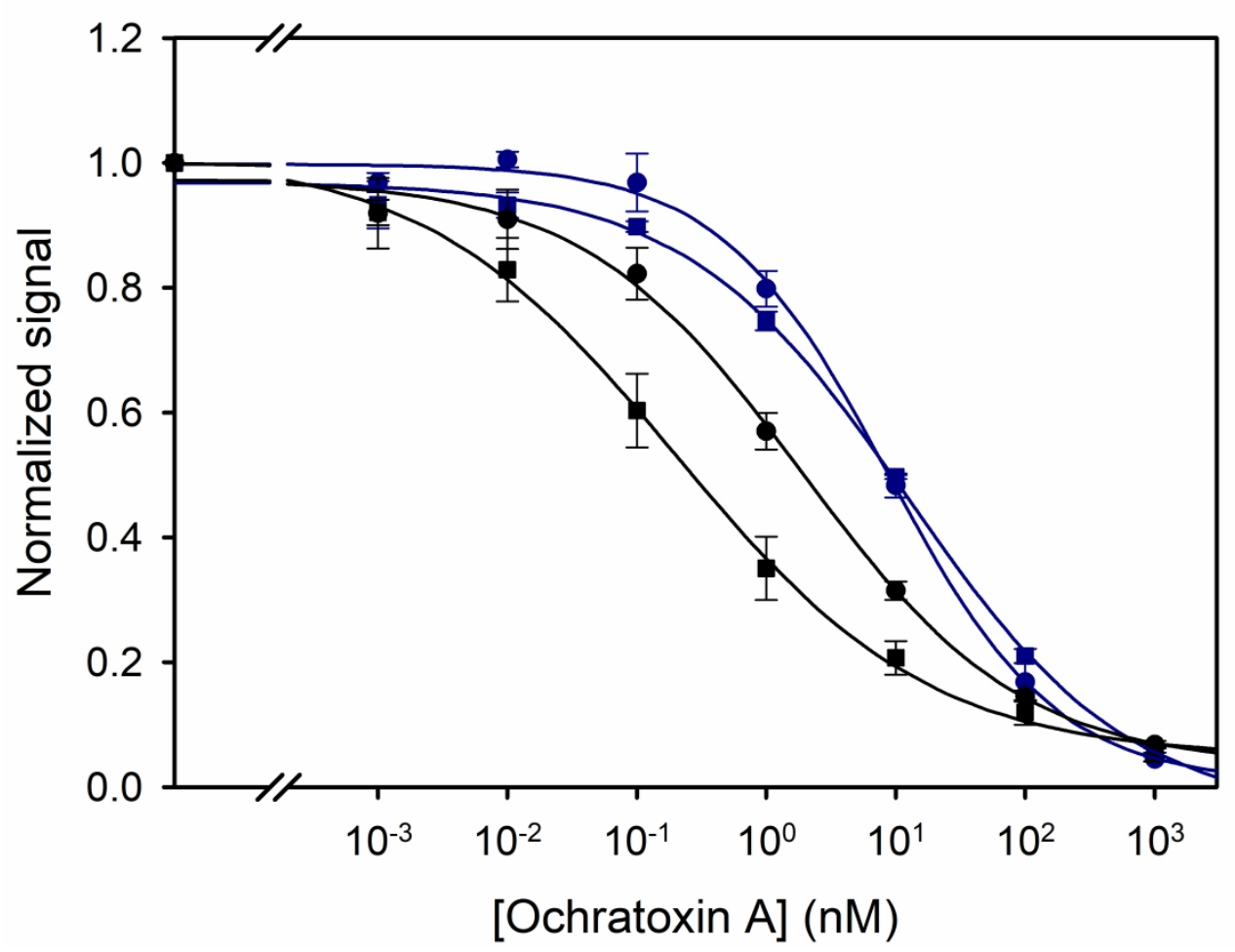

Figure 2. Recognition of OTA by polyclonal antibodies from rabbits immunized with bioconjugate BSA-a (blue lines) and BSA-c (black lines). Each line represents the immune response of a different animal. Experimental points by competitive ELISA are the average of three replicates on different days.

$$
152 \times 122 \mathrm{~mm}(600 \times 600 \text { DPI })
$$

